\title{
Macroscopic description of complex adaptive networks coevolving with dynamic node states
}

\author{
Marc Wiedermann, ${ }^{1,2, *}$ Jonathan F. Donges, ${ }^{1,3}$ Jobst Heitzig, ${ }^{1}$ Wolfgang Lucht, ${ }^{1,4}$ and Jürgen Kurths ${ }^{1,2,5,6}$ \\ ${ }^{1}$ Potsdam Institute for Climate Impact Research, P. O. Box 6012 03, 14412 Potsdam, Germany, EU \\ ${ }^{2}$ Department of Physics, Humboldt University, Newtonstr. 15, 12489 Berlin, Germany, EU \\ ${ }^{3}$ Stockholm Resilience Centre, Stockholm University, Kräftriket 2B, 11419 Stockholm, Sweden, EU \\ ${ }^{4}$ Department of Geography, Humboldt University, Rudower Chaussee 16, 12489 Berlin, Germany, EU \\ ${ }^{5}$ Institute for Complex Systems and Mathematical Biology, University of Aberdeen, Aberdeen AB24 3FX, United Kingdom, EU \\ ${ }^{6}$ Department of Control Theory, Nizhny Novgorod State University, Gagarin Avenue 23, 606950 Nizhny Novgorod, Russia
}

(Received 16 December 2014; published 7 May 2015)

\begin{abstract}
In many real-world complex systems, the time evolution of the network's structure and the dynamic state of its nodes are closely entangled. Here we study opinion formation and imitation on an adaptive complex network which is dependent on the individual dynamic state of each node and vice versa to model the coevolution of renewable resources with the dynamics of harvesting agents on a social network. The adaptive voter model is coupled to a set of identical logistic growth models and we mainly find that, in such systems, the rate of interactions between nodes as well as the adaptive rewiring probability are crucial parameters for controlling the sustainability of the system's equilibrium state. We derive a macroscopic description of the system in terms of ordinary differential equations which provides a general framework to model and quantify the influence of single node dynamics on the macroscopic state of the network. The thus obtained framework is applicable to many fields of study, such as epidemic spreading, opinion formation, or socioecological modeling.
\end{abstract}

DOI: 10.1103/PhysRevE.91.052801

PACS number(s): 89.75.Hc, 87.23.Ge, 89.65.-s, 89.75.Fb

\section{INTRODUCTION}

Complex network theory has proven to be a powerful tool for studying properties, dynamics, and evolution of many real-world complex systems [1,2]. Of particular interest is the ability to investigate adaptive or temporal networks and their respective dynamics [3-5]. Typical processes studied in this field are epidemic spreading [6-8] or opinion formation, e.g., based on the adaptive voter model $[9,10]$. Interactions are modeled by randomly picking a pair of linked nodes and, with fixed probabilities, either changing the state of one of the two nodes or modifying their neighborhood structure by adaptive rewiring. However, recent results have emphasized that opinion formation and imitation processes in fact do not take place with fixed probabilities but can depend on the payoff or performance of different opinion-related choices made by the agents or nodes involved [11-13].

In addition to the structure and dynamics of networks there have been a variety of studies on the dynamics on networks, where nodes in the network represent individual dynamical systems and links indicate directed or symmetric interactions between them $[14,15]$. It has been suggested that the interplay between the dynamics of and on networks should be much more thoroughly investigated, since the dynamics of each of the coupled subsystems is expected to change significantly when compared to their autonomous time evolution [3].

In this work, we propose a model that combines both aspects. For this purpose we refine the adaptive voter model so there is no fixed probability for pairs of nodes to either imitate each other's opinion or adaptively rewire their acquaintance structure. Instead, each node also represents a dynamical system which, for illustration, is chosen here to be simple and easily understood if treated in an isolated fashion. In particular,

*marcwie@pik-potsdam.de we choose a logistic growth model, which is a paradigm for the dynamics of a bounded renewable resource [16]. Whenever interactions between nodes take place, the states of the respective dynamical systems are also taken into account. As a consequence, imitation processes depend explicitly on the nodes' states as well as on the current network structure. At the same time each of the nodes' opinions influences a parameter of the local dynamical system.

The proposed model serves as a narrative for possibly emerging dynamics in coevolutionary human-nature interactions [17-19]. It complements conceptual studies on the effects of economic growth on the ecospheric state $[20,21]$ as well as work on resource exploitation models that take into account the coevolution of stylized resource dynamics with a similarly paradigmatic population growth model [22,23]. The proposed model, for the first time, takes into account individual pairwise interactions of agents on a social network when studying the stability and dynamics of such intertwined systems.

So far, in the context of sustainability science [24], studies on the effect of different exploitation strategies on the state of a certain ecospheric component have been carried out by, e.g., studying the extraction of water in rivers by a network of interconnected harvesters [25-27]. However, no systematic analysis of the underlying network structure and resulting dynamics was performed. In addition, no network dynamics, such as adaptation or imitation processes, were included in these studies and the focus was mainly set on studying the state of the ecosphere for different harvesting strategies that were evolving deterministically in order to optimize all harvesters' payoffs.

In contrast, imitation dynamics with high numbers of agents or players have been studied in the context of evolutionary game theory $[12,13,28,29]$. However, in no such cases were the dynamics of resources or other externalities taken into account and, hence, no coevolution of different subsystems has been studied. Here, the proposed model serves to illustrate the rich dynamics that may emerge from the coupling of these 
different subsystems, even though the complexity in each of the subcomponents remains manageable.

After the introduction of all key components and processes constituting the model in Sec. II we perform numerical simulations of the system. In Sec. III we first study the case of a static network where no adaptation is taking place. We find that the system converges into either a state where all logistic growth models, e.g., resources, converge into a state of full depletion or into a state of positive stock. The latter is to be interpreted as the more sustainable and, hence, desired outcome of the model. We uncover that the likelihood to converge into either of the two states is mainly determined by the frequency of interactions between nodes.

In Sec. IV we then study the effect of network adaptation and show that the stability of the system changes in dependence on the choice of the adaptation frequency. Specifically we deduce that for each interaction frequency there exists an appropriate rate of network adaptation such that the system can be guided into a sustainable state.

Finally, we derive a low-dimensional set of rate equations for variables that approximate the model's macroscopic state in Sec. III B for the static and in Sec. IV for the adaptive case. These equations are generally applicable to any study of opinion formation or spreading if the probabilities of changes in node states by imitation are appropriately chosen. Finally, conclusion are drawn in Sec. V.

\section{MODEL DESCRIPTION}

Assume a temporal network $G[V, L(t)]$ consisting of a fixed set of $N$ nodes $V=\left\{v_{1}, v_{2}, \ldots, v_{N}\right\}$ and an evolving set of links $L(t)$. It is represented by the time-dependent adjacency matrix $A(t)$. Each node $v_{i}$ represents a renewable resource stock $s_{i}(t)$ that obeys a logistic growth model and is harvested with an effort level $E_{i}(t)$ [16],

$$
\frac{d}{d t} s_{i}(t)=a_{i} s_{i}(t)\left(1-s_{i}(t) / K_{i}\right)-q_{i} s_{i}(t) E_{i}(t) .
$$

For this study, we set the growth rates $a_{i}=1$, capacities $K_{i}=1$, and catch coefficients $q_{i}=1$ for all $i=1, \ldots, N$ and measure the time and stocks in dimensionless quantities. Treating all stocks $s_{i}$ as evolving under identical conditions is a strong assumption of the model but allows us to solely focus on the interplay between network and stock dynamics and its dependence on a few key parameters.

The effort is a time-dependent quantity assigned to each node $v_{i}$ which defines its current behavioral pattern and changes through imitation of other nodes. On the one hand, nodes can adopt a high effort level $E_{+}>1$, causing each stock to converge to a stable fixed point $s_{+}=0$, implying full depletion of the resource. Alternatively, nodes can choose a low effort level $E_{-} \in(0,1)$ providing less harvest per unit time initially but avoiding depletion of the resource stocks since each individual stock $s_{i}$ then converges to a stable positive fixed point $s_{-}=1-E_{-}>0$. The two possible choices of effort level, $E_{-}$(low) and $E_{+}$(high), are the same for all nodes and are parameterized by $\Delta E \in(0,1)$ such that $E_{-}=1-\Delta E$ and $E_{+}=1+\Delta E$. At each time $t$ there are $N_{-}(t)$ nodes with $E_{i}(t)=E_{-}$and $N_{+}(t)=N-N_{-}(t)$ nodes with $E_{i}(t)=E_{+}$. The effort then yields for each node $v_{i}$ an individual harvest $h_{i}(t)=s_{i}(t) E_{i}(t)$, which constitutes the second term in Eq. (1).
From now onward we omit the explicit time dependence of the stocks $s_{i}$, efforts $E_{i}$, the adjacency matrix $A$, and the number of low- and high-effort nodes $N_{ \pm}$in our notation.

Initially, for each node $v_{i}$, an individual waiting time $T_{i}$ is drawn at random from a Poissonian distribution with density

$$
p\left(T_{i}\right)=T^{-1} \exp \left(-T_{i} / T\right),
$$

which is a typical choice for modeling interaction rates in social systems [30]. $T$ denotes the expected waiting time between two interactions initiated by the same node $v_{i}$. Starting from this:

(i) The system as given in Eq. (1) is integrated forward in time for the minimum of all current waiting times $T_{i}$. Then, for the corresponding node $v_{i}$ (with the smallest $T_{i}$ ), a neighboring node $v_{j}$ is drawn uniformly at random.

(ii) If the efforts $E_{i}$ and $E_{j}$ of $v_{i}$ and $v_{j}$ differ:

(a) With a rewiring probability $0 \leqslant \phi \leqslant 1, v_{i}$ breaks its link with $v_{j}$ such that $A_{i j}=1$ becomes $A_{i j}=0$. Then a new link between $v_{i}$ and another randomly drawn node $v_{k}$ with the same effort level $\left(E_{i}=E_{k}\right)$ is established such that $A_{i k}=0$ becomes $A_{i k}=1$. This network adaptation process mimics generally observed tendencies to form clusters of individuals with similar behavior or social traits. Note that, in contrast to earlier work, rewiring only takes place if a randomly drawn neighbor $v_{j}$ of $v_{i}$ shows a different effort, e.g., behavioral pattern [10].

(b) If $v_{i}$ does not adapt its neighborhood, imitation may happen instead (with probability $1-\phi$ ). The difference in current harvest $\Delta h_{i j}=h_{j}-h_{i}$ is computed and the node $v_{i}$ imitates the current effort level of $v_{j}$ with a probability given by a sigmoidal function $p\left(E_{i} \rightarrow E_{j}\right)=p\left(\Delta h_{i j}\right)$ which generally is required to be monotonic and continuously differentiable. Additionally, it must fulfill $p\left(\Delta h_{i j}\right) \rightarrow 0$ for $\Delta h_{i j} \rightarrow-\infty$ and $p\left(\Delta h_{i j}\right) \rightarrow 1$ for $\Delta h_{i j} \rightarrow \infty$ and $p(0)=0.5$. This represents the increasing likelihood of imitation processes to take place with an increase in the expected payoff difference [13]. For our model we set $p\left(E_{i} \rightarrow E_{j}\right)=0.5\left(\tanh \Delta h_{i j}+1\right)$ which obeys all of the above requirements.

(iii) A new waiting time $T_{i}$ is drawn at random for $v_{i}$ according to Eq. (2) and step (i) is repeated as long as the model has not reached a steady state.

(iv) The model reaches (with probability one) a steady state at some time $t_{f}$ when the network divides into one or more components in each of which only one choice of effort level is left.

Initially, the two possible effort levels are distributed evenly among the nodes with ratios $n_{-}(0)=N_{-}(0) / N=n_{+}(0)=$ $N_{+}(0) / N=0.5$. Initial stocks are set to $s_{i}(0)=1$ for all $i=1, \ldots, N$. In the following, we consider initially ErdôsRényi random networks with $N=400$ nodes and a linking probability of $\rho=\bar{k} /(N-1)$, where $\bar{k}=20$ is the average degree of nodes in the network.

\section{STATIC NETWORK}

We first study the case of a static network structure with $\phi=$ 0 [hence, modeling step (ii)(a) is not implemented at first] and simulate the model numerically for different combinations of $T$ and $\Delta E$. From this, we derive a macroscopic approximation 
of the model constituted from a set of three coupled differential equations and show its good agreement with the numerical results.

\section{A. Numerical simulations}

Numerical simulations for different combinations of $T$ and $\Delta E$ provide insights into the system's dynamics. Figure 1(a) shows the fraction $f_{-}\left(t_{f}\right)$ of model runs that converge to a state where all nodes show a low effort $E_{i}\left(t_{f}\right)=E_{-} \forall i=1, \ldots, N$ (using an ensemble of $n=500$ simulations). For small $T$ (fast interactions) there is a high probability for the system to converge to a state where only nodes with a high effort level $E_{+}$are present. In this case all resource stocks converge to the stable fixed point $s_{+}=0$ and become fully depleted. With increasing $T$, the system's expected equilibrium state undergoes a phase transition in $f_{-}\left(t_{f}\right)$. For sufficiently large $T$ (slow interactions), the system is likely to converge to a state where all nodes adopt the effort level $E_{-}$and all stocks converge to a stable fixed point $s_{-}=1-E_{-}>0$. This indicates that the rate of interactions between nodes plays a crucial role in determining the system's expected equilibrium state.

The resulting dynamics can be qualitatively understood by considering the limiting cases of $T \rightarrow 0$ and $T \rightarrow \infty$. In the first case, interactions between nodes are expected to happen very fast. Given that initially all stocks carry the same value $s_{i}(0)=s_{0}$ we expect that for $t \ll 1$ the harvest $h_{-}\left(h_{+}\right)$of nodes with low (high) effort follows $h_{-}(t \ll 1) \propto$ $E_{-} s_{0}\left[h_{+}(t \ll 1) \propto E_{+} s_{0}\right]$. This implies that the difference in harvest between the two different types of nodes is expected as $h_{+}(t \ll 1)-h_{-}(t \ll 1) \propto\left(E_{+}-E_{-}\right) s_{0}=2 \Delta E s_{0}$. If interactions happen very fast, the system likely converges into its equilibrium state at $t_{f} \ll 1$. Since in this situation we expect $h_{+}>h_{-}$, nodes with low effort are more likely to imitate the high effort rather than the other way around and, hence, we expect $f_{-}\left(t_{f}\right) \rightarrow 0$ for $T \rightarrow 0$ [as can be seen in Fig. 1(a)].

In contrast, for $T \rightarrow \infty$, we expect updates between nodes to happen preferably at times $t \gg 1$. In this case, the stocks of nodes with high (low) effort can be assumed to have already converged to a fixed point of $s_{+}=0\left(s_{-}=1-E_{+}=\Delta E\right)$
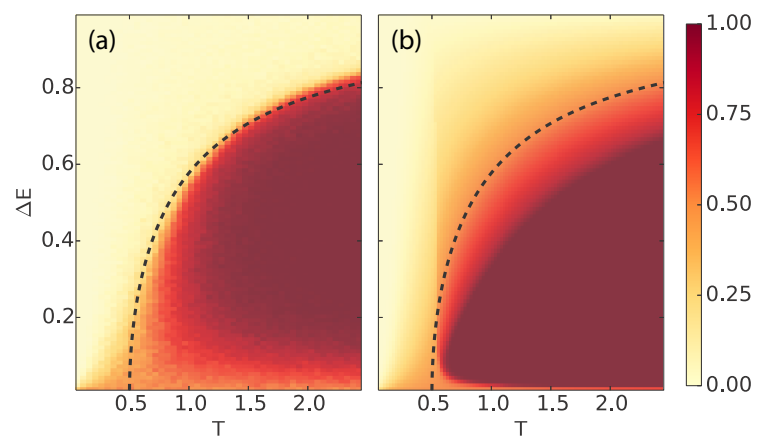

FIG. 1. (Color online) (a) The fraction $f_{-}\left(t_{f}\right)$ of numerical simulations that converge to a state where all nodes show a low effort level $E_{i}\left(t_{f}\right)=E_{-} \forall i=1, \ldots, N$ computed over $n=500$ runs for different choices of $T$ and $\Delta E$ for a static network with $\phi=0$. (b) The value $n_{-0}$ of the stable fixed point for the fraction $n_{-}$of nodes with effort level $E_{-}$computed from Eqs. (16)-(18). The dashed line indicates the critical expected waiting time $T_{c}$ which separates the two regimes (predominance of nodes using $E_{+}$[yellow (light)] and $E_{-}[$red (dark)]. as interactions between nodes start to take place. Hence, the difference in harvest is expected as $h_{-}(t \gg 1)-h_{+}(t \gg 1)=$ $\Delta E-\Delta E^{2}$. Thus, for all $\Delta E \in(0,1)$ the harvest of low-effort nodes exceeds that of nodes with high effort and the system is likely to converge into a state where all nodes show the low effort and, hence, $f_{-}\left(t_{f}\right) \rightarrow 1$ [red (dark) area in Fig. 1(a) for high values of $T]$.

We note that $h_{-}(t \gg 1)-h_{+}(t \gg 1)=\Delta E-\Delta E^{2}$ varies with $\Delta E$. Specifically, in the limiting cases $\Delta E=0$ and $\Delta E=1$ we find that the difference $h_{-}(t \gg 1)-h_{+}(t \gg 1)=$ 0 vanishes and, hence, the system becomes equally likely to converge into either a state with only low-effort nodes or only high-effort nodes present [see lower right corner and the shift of the transition point towards higher $T$ with increasing $\Delta E$ in Fig. 1(a)].

\section{B. Macroscopic approximation}

Abstracting from pairwise microscopic interactions, we now look at the system from a macroscopic point of view. Assuming the network to be large and fully connected at first, the time evolution of the system's state can be characterized by rate equations for three quantities: (1) the fraction of nodes $n_{-}$with effort level $E_{-}$, (2) the mean resource stock $\mu_{-}=\left\langle s_{i} \mid E_{i}=E_{-}\right\rangle_{i}$ of nodes with effort level $E_{-}$, and (3) the mean resource stock $\mu_{+}=\left\langle s_{i} \mid E_{i}=E_{+}\right\rangle_{i}$ of nodes with effort level $E_{+}$. The fraction of nodes $n_{+}$with effort level $E_{+}$ follows from $n_{+}=1-n_{-}$.

The time evolution of $n_{-}$is governed by nodes that change from the low to the high effort level and vice versa. In particular, in the time interval $(t, t+d t)$ an infinitesimal fraction of $d n_{-\rightarrow+}\left(d n_{+\rightarrow-}\right)$ nodes change their effort from $E_{-}\left(E_{+}\right)$to $E_{+}\left(E_{-}\right)$, which decreases (increases) the fraction of nodes with low effort $n_{-}$,

$$
d n_{-}=d n_{+\rightarrow-}-d n_{-\rightarrow+} .
$$

The interactions between nodes that govern the rates of changes in effort are driven by the following quantities:

(1) The expected waiting time $T$ for a node $v_{i}$ to interact with a randomly drawn neighboring node $v_{j}$. Correspondingly, the rate of node interactions is taken to be $\tau=1 / T$.

(2) If a node $v_{i}$ interacts with its neighboring node $v_{j}$, an imitation of effort only takes place if $E_{i} \neq E_{j}$. Hence, for a node $v_{i}$ with $E_{i}=E_{-}\left(E_{i}=E_{+}\right)$there is to define a probability $P_{-}^{+}\left(P_{+}^{-}\right)$that a randomly drawn neighboring node $v_{j}$ has $E_{j}=E_{+}\left(E_{j}=E_{-}\right)$. Since a large fully connected network is assumed, this probability is given exactly by the current fraction $n_{+}\left(n_{-}\right)$of nodes with high (low) effort $E_{+}$ $\left(E_{-}\right)$and, hence, $P_{-}^{+}=n_{+}\left(P_{+}^{-}=n_{-}\right)$.

(3) If a node $v_{i}$ with $E_{i}=E_{-}\left(E_{i}=E_{+}\right)$interacts with a neighboring node $v_{j}$ with $E_{j}=E_{+}\left(E_{j}=E_{-}\right)$, there is a probability $p_{-\rightarrow+}\left(p_{+\rightarrow-}\right)$ that $v_{i}$ takes up the effort level $E_{j}$ of $v_{j}$. This probability is governed by the difference in harvest $\Delta h_{i j}$ between $v_{j}$ and $v_{i}$. For the macroscopic description, the individual pairwise interactions are replaced by aggregated quantities. Therefore $p_{-\rightarrow+}\left(p_{+\rightarrow-}\right)$ is computed as the expected probability for a node $v_{i}$ with low (high) effort to adopt the high (low) effort given that it interacts with a node $v_{j}$ that currently has $E_{j}=E_{+}\left(E_{j}=E_{-}\right)$. This quantity is then dependent on the expected stocks at nodes with low and high effort, which is derived below in detail. 
This yields $d n_{-\rightarrow+}$ and $d n_{+\rightarrow-}$ as the product of all three factors introduced above,

$$
\begin{gathered}
d n_{-\rightarrow+}=n_{-} \tau n_{+} p_{-\rightarrow+} d t, \\
d n_{+\rightarrow-}=n_{+} \tau n_{-} p_{+\rightarrow-} d t, \\
\Rightarrow \frac{d n_{-}}{d t}=\tau n_{-} n_{+}\left(p_{+\rightarrow-}-p_{-\rightarrow+}\right) .
\end{gathered}
$$

The two quantities still remaining to be evaluated are the expected probabilities $p_{+\rightarrow-}\left(p_{-\rightarrow+}\right)$ for nodes with a high (low) effort level to change to the opposite level. It is obtained as the expected probability for nodes in the network to take up its neighbor's effort,

$$
\begin{aligned}
p_{+\rightarrow-} & =\left\langle p\left(E_{j} \rightarrow E_{k}\right) \mid E_{j}=E_{+}, E_{k}=E_{-}\right\rangle_{j, k} \\
& =0.5\left\langle\tanh \left(\Delta h_{j k} \mid E_{j}=E_{+}, E_{k}=E_{-}\right)\right\rangle_{j, k}+0.5 \\
& \cong 0.5\left\langle\Delta h_{j k} \mid E_{j}=E_{+}, E_{k}=E_{-}\right\rangle_{j, k}+0.5 \\
& =0.5\left(E_{-}\left\langle s_{k} \mid E_{k}=E_{-}\right\rangle_{k}-E_{+}\left\langle s_{j} \mid E_{j}=E_{+}\right\rangle_{j}\right)+0.5 \\
& =0.5\left(E_{-} \mu_{-}-E_{+} \mu_{+}\right)+0.5 \\
p_{-\rightarrow+} & =0.5\left(E_{+} \mu_{+}-E_{-} \mu_{-}\right)+0.5 .
\end{aligned}
$$

Here we performed a linear expansion of the hyperbolic tangent, $\tanh x=x+O\left(x^{3}\right)$, assuming that differences in harvest remain small.
The time evolution of either of the two average stocks $\mu_{-}$ and $\mu_{+}$is governed by two terms. First, each individual stock $s_{i}$ follows the logistic growth model and so do the average quantities. Second, the value of each of the two average stocks changes according to the fact that the nodes modify their effort from $E_{-}$to $E_{+}$and vice versa during the time interval $(t, t+$ $d t$ ). This yields

$$
\begin{aligned}
d \mu_{-} & =d\left\langle s_{k} \mid E_{k}=E_{-}\right\rangle_{k} \\
& =\left\langle d s_{k} \mid E_{k}=E_{-}\right\rangle_{k} \\
& =d t\left\langle s_{k}\left(1-s_{k}\right)-E_{k} s_{k} \mid E_{k}=E_{-}\right\rangle_{k}+\delta_{-} \\
& =d t \mu_{-}-d t\left\langle s_{k}^{2} \mid E_{k}=E_{-}\right\rangle_{k}-d t E_{-} \mu_{-}+\delta_{-} \\
& =d t\left(\mu_{-}\left(1-\mu_{-}-E_{-}\right)-\mu_{-}^{(2)}\right)+\delta_{-} \\
d \mu_{+} & =d t\left(\mu_{+}\left(1-\mu_{+}-E_{+}\right)-\mu_{+}^{(2)}\right)+\delta_{+} .
\end{aligned}
$$

Here $\mu_{-}^{(2)}$ and $\mu_{+}^{(2)}$ denote the variances in the two types of stocks. $\delta_{-}\left(\delta_{+}\right)$indicate the net change in the average stock as nodes with high (low) effort change their effort to the opposite choice during $(t, t+d t)$. The fraction of nodes $d n_{+\rightarrow-}\left(d n_{-\rightarrow+}\right)$ that change their effort from $E_{+}$to $E_{-}\left(E_{-}\right.$ to $\left.E_{+}\right)$during $(t, t+d t)$ is assumed to be small compared to the fraction of nodes which already hold the low (high) effort, $d n_{+\rightarrow-} \ll n_{-}\left(d n_{-\rightarrow+} \ll n_{+}\right)$. Hence, the respective contribution to the dynamics of $\mu_{-}\left(\mu_{+}\right)$as nodes change their effort is also assumed to be small, $d n_{+\rightarrow-} \mu_{+} \ll n_{-} \mu_{-}$ $\left(d n_{-\rightarrow+} \mu_{-} \ll n_{+} \mu_{+}\right)$. This allows for a first-order expansion of the stock's time evolution, such that

$$
\begin{aligned}
\mu_{-}+\delta_{-}= & \frac{\left(n_{-}-d n_{-\rightarrow+}\right) \mu_{-}+d n_{+\rightarrow-} \mu_{+}}{n_{-}-d n_{-\rightarrow+}+d n_{+\rightarrow-}} \\
\cong & \left.\frac{\left(n_{-}-d n_{-\rightarrow+}\right) \mu_{-}+d n_{+\rightarrow-} \mu_{+}}{n_{-}-d n_{-\rightarrow+}+d n_{+\rightarrow-}}\right|_{\left(d n_{-\rightarrow+}, d n_{+\rightarrow-}\right)(0,0)} \\
& +\left.\frac{-\mu_{-}\left(n_{-}-d n_{-\rightarrow+}+d n_{+\rightarrow-}\right)+\left(\left(n_{-}-d n_{-\rightarrow+}\right) \mu_{-}+d n_{+\rightarrow-} \mu_{+}\right)}{\left(n_{-}-d n_{-\rightarrow+}+d n_{+\rightarrow-}\right)^{2}}\right|_{\left(d n_{-\rightarrow}, d n_{+\rightarrow-}\right)=(0,0)} d n_{-\rightarrow+} \\
& +\left.\frac{\mu_{+}\left(n_{-}-d n_{-\rightarrow+}+d n_{+\rightarrow-}\right)-\left(\left(n_{-}-d n_{-\rightarrow+}\right) \mu_{-}+d n_{+\rightarrow-} \mu_{+}\right)}{\left(n_{-}-d n_{-\rightarrow+}+d n_{+\rightarrow-}\right)^{2}}\right|_{\left(d n_{-\rightarrow+}, d n_{+\rightarrow-}\right)=(0,0)} d n_{+\rightarrow-} \\
= & \frac{n_{-} \mu_{-}}{n_{-}}+\frac{-\mu_{-} n_{-}+n_{-} \mu_{-}}{n_{-}^{2}} d n_{-\rightarrow+}+\frac{\mu_{+} n_{-}-n_{-} \mu_{-}}{n_{-}^{2}} d n_{+\rightarrow-}=\mu_{-}+\frac{\mu_{+}-\mu_{-}}{n_{-}} d n_{+\rightarrow-} \\
\Rightarrow \delta_{-}= & \left(\mu_{+}-\mu_{-}\right) n_{+} \tau p_{+\rightarrow-} d t \\
\delta_{+}= & \left(\mu_{-}-\mu_{+}\right) n_{-} \tau p_{-\rightarrow+} d t .
\end{aligned}
$$

Putting this back into (9) and (10) yields

$$
\begin{aligned}
& d \mu_{-}=d t\left(\mu_{-}\left(1-\mu_{-}-E_{-}\right)-\mu_{-}^{(2)}\right)+d t\left(\mu_{+}-\mu_{-}\right) n_{+} \tau p_{+\rightarrow-} \\
& d \mu_{+}=d t\left(\mu_{+}\left(1-\mu_{+}-E_{+}\right)-\mu_{+}^{(2)}\right)+d t\left(\mu_{-}-\mu_{+}\right) n_{-} \tau p_{-\rightarrow+} .
\end{aligned}
$$

In the scope of this work, in to order to close the set of equations that describe the systems dynamics, we assume the respective variances $\mu_{-}^{(2)}$ and $\mu_{+}^{(2)}$ to vanish. Taking into account higher moments in the dynamics of the stocks and investigate its influence on the resulting fixed points remains as a task for future research.

In summary, we find a set of three coupled ordinary differential equations that define the time evolution of the static network model:

$$
\frac{d n_{-}}{d t}=\tau n_{+} n_{-}\left(p_{+\rightarrow-}-p_{-\rightarrow+}\right)
$$




$$
\begin{aligned}
& \frac{d \mu_{-}}{d t}=\mu_{-}\left(1-\mu_{-}-E_{-}\right)+\tau\left(\mu_{+}-\mu_{-}\right) n_{+} p_{+\rightarrow-} \\
& \frac{d \mu_{+}}{d t}=\mu_{+}\left(1-\mu_{+}-E_{+}\right)+\tau\left(\mu_{-}-\mu_{+}\right) n_{-} p_{-\rightarrow+} .
\end{aligned}
$$

\section{Fixed points and stability}

We obtain all fixed points $P_{i}=\left(n_{-0}, \mu_{-0}, \mu_{+0}\right)$ of the dynamical system given in Eqs. (16)-(18) as:

$$
\begin{aligned}
P_{1}= & \left(n_{-0}=0, \mu_{-0}=\frac{1-E_{-}-0.5 \tau}{1+0.5 \tau E_{-}}, \mu_{+0}=0\right), \\
P_{2}= & \left(n_{-0}=1, \mu_{-0}=0, \mu_{+0}=\frac{1-E_{+}-0.5 \tau}{1+0.5 \tau E_{+}}\right), \\
P_{3}= & {\left[n_{-0}=\frac{2\left(E_{-} \frac{1-0.5 \tau}{E_{-}+E_{+}}+E_{+}-1\right)}{\tau\left(\frac{E_{+}}{E_{-}}-1\right)}, \mu_{-0}=E_{+} \frac{1-0.5 \tau}{E_{-}+E_{+}}, \mu_{+0}=E_{-} \frac{1-0.5 \tau}{E_{-}+E_{+}}\right], } \\
P_{4}= & {\left[n_{-0}=1, \mu_{-0}=1-E_{-}, \mu_{+0}=\frac{-b}{2 a}+\sqrt{\left(\frac{b}{2 a}\right)^{2}+\frac{c}{a}}\right], } \\
P_{5}= & {\left[n_{-0}=1, \mu_{-0}=1-E_{-}, \mu_{+0}=\frac{-b}{2 a}-\sqrt{\left(\frac{b}{2 a}\right)^{2}+\frac{c}{a}}\right], } \\
a= & 0.5\left(-2-E_{+} \tau\right) \\
b= & 1-E_{+}+0.5 \tau\left[\left(1-E_{-}\right) E_{+}+E_{-}-E_{-}^{2}-1\right] \\
c= & 0.5 \tau\left(1-E_{-}\right)\left(E_{-}-E_{-}^{2}-1\right) .
\end{aligned}
$$

In addition, there exists a manifold which also satisfies $\frac{d n_{-}}{d t}=\frac{d \mu_{-}}{d t}=\frac{d \mu_{+}}{d t}=0$ and is defined by

$$
P_{\alpha}=\left(n_{-0}=\alpha, \mu_{-0}=0, \mu_{+0}=0\right), \alpha \in[0,1] .
$$

For all fixed points given above we compute the largest eigenvalue $\lambda_{1}$ of the corresponding Jacobian matrix evaluated at the respective point. Only the two fixed points $P_{3}$ and $P_{4}$ have a negative largest eigenvalue $\lambda_{1}<0$ and, hence, are stable for choices of parameters $\Delta E$ and $T>0.5$ (note that, again, $E_{-}=1-\Delta E, E_{+}=1+\Delta E$, and $\tau=1 / T$ ) (Fig. 2).

To investigate the system's dynamics in the regime $T<0.5$, the stability on the one-dimensional manifold defined by all points that fulfill Eq. (24) is assessed. Analytically computing the three eigenvalues of the Jacobian matrix on the manifold as a function of the parameter $\alpha$ yields

$$
\begin{aligned}
\lambda_{0} & =0 \\
\lambda_{ \pm}(\alpha) & =1-\frac{E_{+}+E_{-}}{2}-\frac{\tau}{4} \pm \frac{1}{2} \sqrt{2 \alpha E_{+} \tau-2 \alpha E_{-} \tau+E_{+}^{2}-2 E_{+} E_{-}-E_{+} \tau+E_{-}^{2}+E_{-} \tau+\frac{\tau^{2}}{4} .}
\end{aligned}
$$

A first observation is that $\lambda_{+}(\alpha) \geqslant \lambda_{-}(\alpha)$ holds. Since $\lambda_{0}=0$, it is obvious that not all eigenvalues can be negative. However, if $\lambda_{0}=0$ is the largest eigenvalue of the system, all choices of $\alpha$ for which $\lambda_{+}(\alpha) \leqslant \lambda_{0}$ define a center manifold,

$$
\lambda_{+}(\alpha) \leqslant 0 \quad \text { if } \quad \alpha \leqslant \frac{1}{2}-T \Delta E
$$

Hence,

$$
\begin{aligned}
v(\alpha) & =\left(n_{-0}=\alpha, \mu_{-0}=0, \mu_{+0}=0\right) \\
\alpha & \in\left[0, \frac{1}{2}-T \Delta E\right]
\end{aligned}
$$

defines a center manifold where the system's stability cannot be assessed by linear stability analysis. A detailed study of the system's stability in this regime is beyond the scope of this work and not necessarily needed to understand the general dynamics of the macroscopic description proposed here. Numerically integrating the system for choices of parameters taken from the center manifold, however, reveals good agreement between the microscopic and macroscopic model representation (Fig. 1). An investigation by means of a higher-order stability analysis might yield further insights into the processes that cause both resource stocks $\mu_{-0}=\mu_{+0}=0$ to be fully depleted in the regime of the center manifold. 

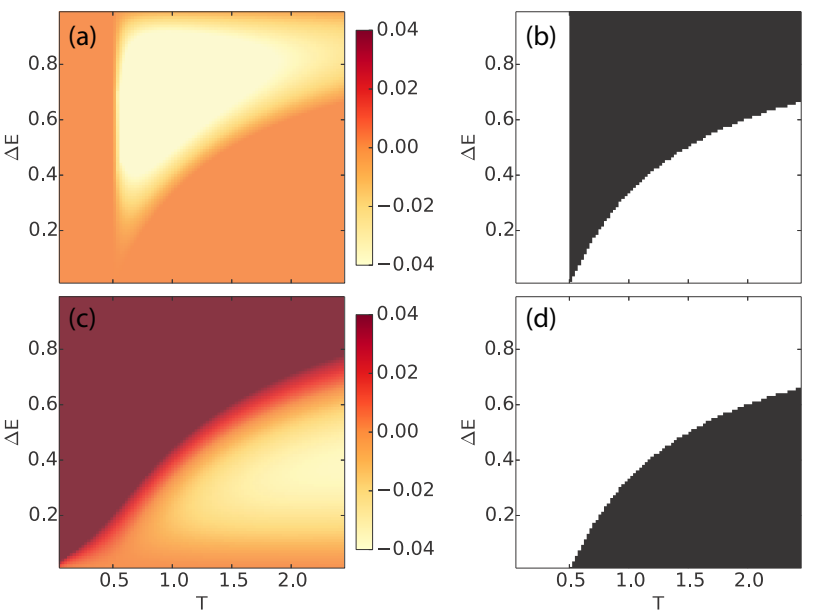

FIG. 2. (Color online) The largest eigenvalue $\lambda_{1}$ for the two fixed points $P_{3}$ (a) and $P_{4}$ (c) [see also Eqs. (21) and (22)] depending on $\Delta E$ and $T$. The black area in (b) indicates the domain in parameter space for which $\lambda_{1}$ computed for $P_{3}$ is negative and, hence, $P_{3}$ is stable. (d) shows the same properties for $P_{4}$. The regimes for which either of the two fixed points is stable are complementary. Further it should be noted that for $T<0.5$ neither of the two fixed points is stable, but the center manifold as given in Eq. (28) exists in this regime.

In conclusion, we note that for each choice of parameters only one of the fixed points $P_{3}$ and $P_{4}$ can be the unique stable fixed point of the system (Fig. 2). Figure 1(b) displays the value of the stable fixed point's $n_{-0}$ component as a function of $T$ and $\Delta E$. The results are in good agreement with the numerical findings [Fig. 1(a)]. Due to the first-order approximation, the transition from a predominance of nodes with $E_{+}$to nodes with $E_{-}$with increasing $T$ is not as sharp as for the numerical simulations. However, a good estimate for the critical value $T_{c}$ of $T$ at which the transition takes place can be found by setting $n_{-0}\left(T_{c}\right)=0.5$ in Eq. (21) which yields $T_{c}(\Delta E)=\frac{1+\Delta E^{2}}{2-2 \Delta E^{2}}$ (dashed line in Fig. 1).

\section{ADAPTIVE NETWORK}

In the following, we consider additionally network adaptation processes with $\phi>0$ [hence, modeling steps (ii)(a) and (ii)(b) both take place with a relative frequency depending on the rewiring probability $\phi]$. For all results presented from here onward, the two available choices of effort levels are fixed by setting $\Delta E=0.5$.

\section{A. Numerical simulations}

Numerical simulations with the same initial conditions as in the static case for different combinations of $\phi$ and $T$ reveal a division of the parameter space into regimes of different expected outcomes as the model reaches its steady state [Fig. 3(a)]. In contrast to the static case nodes no longer necessarily all carry the same effort as the model reaches its equilibrium state, due to the possibility for the network to fragment into smaller components. Hence, from now on $f_{-}\left(t_{f}\right)$ denotes the mean fraction of nodes with effort level $E_{-}$as the model reaches consensus. As for $\phi=0$, fast interactions (i.e.,
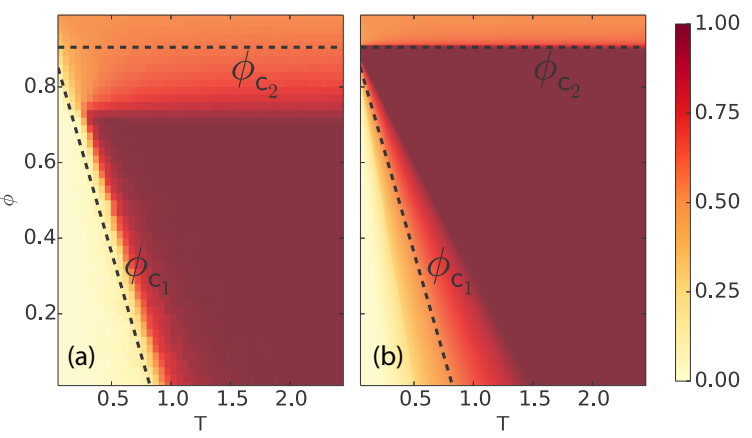

FIG. 3. (Color online) (a) Mean fraction of nodes $f_{-}\left(t_{f}\right)$ with effort level $E_{-}=1-\Delta E=0.5$ for different choices of $T$ and $\phi$ obtained from an ensemble of $n=500$ simulations as the system reaches its steady state. (b) Value of the stable fixed point for the fraction of nodes with effort level $E_{-}$computed from the set of differential equations (59)-(63).

low values of $T$ ) lead to a large fraction of nodes carrying $E_{+}$. The transition between the two behavioral patterns with increasing $T$ remains sharp. However, depending on the choice of $\phi$, the value of the critical waiting time $T_{c}$, at which the system transfers from a state with a predominance of nodes with low effort to a state with a predominance of nodes with high effort decreases with increasing $\phi$. Conversely, this implies that for all $T \gtrsim 0.3$ there is an appropriate choice of $\phi \in\left[\phi_{c_{1}}, \phi_{c_{2}}\right]$ so that all nodes are likely to adopt the effort level $E_{-}$. In the limiting case of $\phi=1$ the expected fraction of nodes with $E_{-}$equals the initial fraction $n_{-}(0)=0.5$ for all choices of $T$ due to the network's fragmentation into components of nodes sharing the same effort.

\section{B. Macroscopic approximation}

The macroscopic approximations (16)-(18) can be extended to also include the effects of network rewiring. For this, we introduce two additional variables describing the macroscopic state of the network. The time evolution of the fraction of nodes $n_{-}$with low effort is recalled [analogously to Eq. (6)] as

$$
\frac{d n_{-}}{d t}=\tau\left(n_{+} P_{+}^{-} p_{+\rightarrow-}-n_{-} P_{-}^{+} p_{-\rightarrow+}\right) .
$$

Given that a node $v_{i}$ initializes an interaction and the randomly drawn neighboring node $v_{j}$ employs a different effort, $E_{i} \neq$ $E_{j}$, there exists the adaptive rewiring probability $\phi \in[0,1]$ for $v_{i}$ to break its connection with $v_{j}$ and establish a link with another randomly drawn node $v_{k}$ in the network that is employing the same effort as $v_{i}\left(E_{k}=E_{i}\right)$ and is not yet connected to node $v_{i}$. With probability $1-\phi$, imitation of efforts takes place which has already been implemented in the macroscopic description of the static network. To account for the adaptive rewiring process, the interaction rate $\tau$ needs to be refined such that it no longer represents the rate of node interactions alone, but the rate of interactions which lead to imitation,

$$
\tau=\frac{1-\phi}{T} .
$$


Likewise the ratio $\rho$ of all node interactions that lead to adaptive rewiring needs to be defined. Since each node is expected to interact at a rate $1 / T$ it follows that

$$
\rho=\frac{\phi}{T} .
$$

For adaptive rewiring to take place, the network cannot be fully connected. Therefore, the previous definitions of $P_{+}^{-}=n_{-}$and $P_{-}^{+}=n_{+}$for two nodes of different effort to interact no longer hold for the derivations to be performed here.

The total number of $M$ links in the network splits into $M_{-}$ $\left(M_{+}\right)$links connecting two nodes with low (high) effort and $M_{+-}$links connecting two nodes of different efforts, such that

$$
\begin{gathered}
M=\frac{N \bar{k}}{2}=M_{-}+M_{+}+M_{+-} \\
\Rightarrow \frac{d M}{d t}=\frac{d M_{-}}{d t}+\frac{d M_{+}}{d t}+\frac{d M_{+-}}{d t}=0 .
\end{gathered}
$$

Additionally, let

$$
K_{-}^{-}=\frac{2 M_{-}}{N_{-}}
$$

denote for nodes with low effort the average number of neighbors with the same effort. Likewise,

$$
K_{-}^{+}=\frac{M_{+-}}{N_{-}}
$$

represents for nodes with low effort the average number of neighbors with high effort. These two quantities constitute the average degree of nodes with low effort as

$$
K_{-}=K_{-}^{-}+K_{-}^{+}=\frac{M_{+-}+2 M_{-}}{N_{-}} .
$$

Likewise, the average degree $K_{+}$of nodes with high effort is obtained from

$$
\begin{aligned}
& K_{+}^{+}=\frac{2 M_{+}}{N_{+}}, \\
& K_{+}^{-}=\frac{M_{+-}}{N_{+}}, \\
& K_{+}=\frac{M_{+-}+2 M_{+}}{N_{+}} .
\end{aligned}
$$

For a node $v_{i}$ currently having a low effort $E_{i}=E_{-}$the probability $P_{-}^{+}\left(v_{i}\right)$ to draw a neighbor $v_{j}$ with different effort at random is given as

$$
P_{-}^{+}\left(v_{i}\right)=\frac{k_{-}^{+}\left(v_{i}\right)}{k\left(v_{i}\right)},
$$

where $k_{-}^{+}\left(v_{i}\right)$ is the number of neighbors of node $v_{i}$ that employ the high effort and $k\left(v_{i}\right)$ denotes the degree of node $v_{i}$. Since for the macroscopic description the pairwise microscopic interactions between nodes are approximated by the average dynamics, we compute the average probability $P_{-}^{+}$for a node $v_{i}$ with low effort to interact with a node employing the high effort. Since the network is initialized as an Erdős-Rényi random network and it is further equally likely for all nodes with the same effort to connect to or disconnect from other nodes by random rewiring, we perform a heterogeneous mean-field approximation and assume the degree $k\left(v_{i}\right)$ to be the same for all nodes with low effort, $k\left(v_{i}\right)=K_{-} \forall i \in\left\{1, \ldots, N \mid E_{i}=E_{-}\right\}[31,32]$. Thus

$$
\begin{aligned}
P_{-}^{+} & =\left\langle P_{-}^{+}\left(v_{i}\right) \mid E_{i}=E_{-}\right\rangle_{i}=\left\langle\frac{k_{-}^{+}\left(v_{i}\right)}{k\left(v_{i}\right)} \mid E_{i}=E_{-}\right\rangle_{i} \\
& =\left\langle\frac{k_{-}^{+}\left(v_{i}\right)}{K_{-}} \mid E_{i}=E_{-}\right\rangle_{i}=\frac{K_{-}^{+}}{K_{-}} \\
& =\frac{M_{+-}}{2 M_{-}+M_{+-}} .
\end{aligned}
$$

Instead of the actual number of $M$ links in the network we define the corresponding per node link density

$$
\begin{aligned}
m & =\frac{M}{N}=\frac{M_{+-}}{N}+\frac{M_{-}}{N}+\frac{M_{+}}{N} \\
& =\frac{\bar{k}}{2}=m_{+-}+m_{-}+m_{+},
\end{aligned}
$$

which is independent of the number of nodes $N . \bar{k}$ denotes the average degree of nodes in the network, which is set to $\bar{k}=20$ in accordance with the numerical simulations. The probability for a node with low (high) effort to interact with a node of high (low) effort is then given by

$$
\begin{aligned}
& P_{-}^{+}=\frac{m_{+-}}{2 m_{-}+m_{+-}}, \\
& P_{+}^{-}=\frac{m_{+-}}{2 m_{+}+m_{+-}},
\end{aligned}
$$

and is fully determined by the per node densities of links $m_{+-}$, $m_{+}$, and $m_{-}$.

Generally, the time evolution of the total number of links between nodes of low effort is governed by imitation and adaptation. First, we focus on the process of adaptation. Since links between nodes of the same effort can only be established but not removed via the process of adaptation, the contribution of this process to the total number of links between low-effort nodes $M_{-}$only causes it to increase. This positive contribution is

$$
\frac{d M_{-}}{d t} \sim \rho N_{-} P_{-}^{+}
$$

and is explained as follows: In each time interval $(t, t+d t)$ there is a total number of $N_{-}$nodes which with probability $\rho$ initiate an interaction that leads to adaptive rewiring. Adaptive rewiring then takes place if a randomly drawn neighbor $v_{j}$ of the considered node $v_{i}$ employs a high effort. As defined above, this happens with probability $P_{-}^{+}$.

The second contribution to the time evolution of $M_{-}$is given by imitation, which takes place at rate $\tau$. Generally, there is one term causing an increase in links between nodes with low effort and one term causing its decrease. First, assume a node $v_{i}$ with $E_{i}=E_{+}$to imitate the low effort $E_{-}$from one of its neighboring nodes $v_{j}$ with $E_{j}=E_{-}$. The number of links between nodes of low effort then increases by the number $k_{+}^{-}\left(v_{i}\right)$ of all neighbors of node $v_{i}$ that employ the low effort (Fig. 4). Again, by performing a heterogeneous meanfield approximation and assuming the number of neighbors for individual nodes to be represented by the respective average 


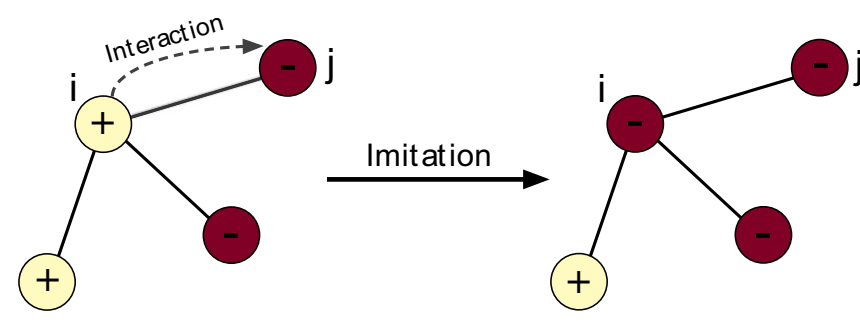

FIG. 4. (Color online) Illustration of the influence of the imitation of effort on the different numbers of link types in the network. A node $v_{i}$ with the high effort $E_{i}=E_{+}$[indicated in yellow (light)] interacts with a node $v_{j}$ with low effort $E_{j}=E_{-}$[red (dark)]. Node $v_{i}$ may then imitate the effort of node $v_{j}, E_{i} \rightarrow E_{-}$. The number of links between nodes with low (high) effort $M_{-}\left(M_{+}\right)$then increases (decreases) by the number $k_{+}^{-}\left(v_{i}\right)\left[k_{+}^{+}\left(v_{i}\right)\right]$ of neighbors of $v_{i}$ that show the low (high) effort.

number of neighbors, we set

$$
k_{+}^{-}\left(v_{j}\right)=K_{+}^{-}=\frac{M_{+-}}{N_{+}} .
$$

Now, each of the $N_{+}$nodes with high effort interacts with a node of low effort with probability $P_{+}^{-}$at rate $\tau$. Then, with probability $p_{+\rightarrow-}$, a node with high effort takes up the low effort. This causes the number of links between pairs of nodes with low effort to increase by the number of neighbors with low effort of the formerly high-effort node,

$$
\frac{d M_{-}}{d t} \sim \tau N_{+} P_{+}^{-} p_{+\rightarrow-} K_{+}^{-} .
$$

A third term that governs the time evolution of $M_{-}$is given by its decrease caused by nodes with low effort that imitate the high effort. If a node $v_{i}$ with the low effort $E_{i}=E_{-}$interacts with a node $v_{j}$ having the high effort $E_{j}=E_{+}$and $v_{i}$ then imitates the effort of $v_{j}$, the total number of links connecting two nodes with low effort decreases by the number of $v_{i}$ 's neighbors $v_{k}$ that are showing the low effort $E_{k}=E_{-}$as well. Following from an analogous argument as given above, this number is given by $k_{-}^{-}\left(v_{i}\right)$. Again we assume the number of neighbors $v_{k}$ with $E_{k}=E_{-}$of a node $v_{i}$ with $E_{i}=E_{-}$to be approximated by its average,

$$
k_{-}^{-}\left(v_{j}\right)=K_{-}^{-}=\frac{2 M_{-}}{N_{-}} .
$$

With rate $\tau$ each of the $N_{-}$nodes with low effort interacts with a node showing the high effort $E_{+}$with probability $P_{-}^{+}$. With probability $p_{-\rightarrow+}$ a node with low effort imitates the high effort which causes a decrease in $M_{-}$by the average number of low-effort neighbors $K_{-}^{-}$of the node that is imitating the high effort,

$$
\frac{d M_{-}}{d t} \sim-\tau N_{-} P_{-}^{+} p_{-\rightarrow+} K_{-}^{-} .
$$

Putting together Eqs. (45), (47), and (49) gives the time evolution of the number of links between nodes of low effort as

$$
\begin{aligned}
\frac{d M_{-}}{d t}= & \tau\left(N_{+} P_{+}^{-} p_{+\rightarrow-} K_{+}^{-}-N_{-} P_{-}^{+} p_{-\rightarrow+} K_{-}^{-}\right) \\
& +\rho N_{-} P_{-}^{+}
\end{aligned}
$$

Plugging the definitions of $K_{-}^{-}$[Eq. (34)] and $K_{+}^{-}$[Eq. (38)] into Eq. (50) and normalizing with the total number of nodes $N$ yields the time evolution of the per node density of links between nodes of low effort,

$$
\frac{d m_{-}}{d t}=\tau\left(P_{+}^{-} p_{+\rightarrow-} m_{+-}-2 P_{-}^{+} p_{-\rightarrow+} m_{-}\right)+\rho n_{-} P_{-}^{+},
$$

which is again independent of $N$. Due to the symmetry of the system, the time evolution of the per node density $m_{+}$of links between nodes with high effort then immediately follows as

$$
\frac{d m_{+}}{d t}=\tau\left(P_{-}^{+} p_{-\rightarrow+} m_{+-}-2 P_{+}^{-} p_{+\rightarrow-} m_{+}\right)+\rho n_{+} P_{+}^{-} .
$$

For the time evolution of the average stock of nodes with low and high effort $\mu_{-}$and $\mu_{+}$we already found in Eqs. (9) and (10) that

$$
\begin{aligned}
& d \mu_{-}=d t\left(\mu_{-}\left(1-\mu_{-}-E_{-}\right)-\mu_{-}^{(2)}\right)+\delta_{-}, \\
& d \mu_{+}=d t\left(\mu_{+}\left(1-\mu_{+}-E_{+}\right)-\mu_{+}^{(2)}\right)+\delta_{+} .
\end{aligned}
$$

The general forms of $\delta_{-}$and $\delta_{+}$are [see Eq. (12) and (13)]

$$
\begin{aligned}
& \delta_{-}=\frac{\mu_{+}-\mu_{-}}{n_{-}} d n_{+\rightarrow-}, \\
& \delta_{+}=\frac{\mu_{-}-\mu_{+}}{n_{+}} d n_{-\rightarrow+} .
\end{aligned}
$$

For the case of an adaptive network, $d n_{+\rightarrow-}\left(d n_{-\rightarrow+}\right)$ is given by the first (second) term in Eq. (29):

$$
\begin{aligned}
& \delta_{-}=\frac{\mu_{+}-\mu_{-}}{n_{-}} \tau n_{+} P_{+}^{-} p_{+\rightarrow-}, \\
& \delta_{+}=\frac{\mu_{-}-\mu_{+}}{n_{+}} \tau n_{-} P_{-}^{+} p_{-\rightarrow+},
\end{aligned}
$$

with the probabilities $P_{-}^{+}$and $P_{+}^{-}$[Eqs. (43) and (44)] as defined above and $p_{+\rightarrow-}$ and $p_{-\rightarrow+}$ being the same as for the static model [Eqs. (7) and (8)].

To summarize, the set of five coupled differential equations that represent the adaptive network model's macroscopic dynamics is given as

$$
\begin{aligned}
\frac{d n_{-}}{d t} & =\tau\left(n_{+} P_{+}^{-} p_{+\rightarrow-}-n_{-} P_{-}^{+} p_{-\rightarrow+}\right) \\
\frac{d m_{-}}{d t} & =\tau\left(P_{+}^{-} p_{+\rightarrow-} m_{+-}-2 P_{-}^{+} p_{-\rightarrow+} m_{-}\right)+\rho n_{-} P_{-}^{+}
\end{aligned}
$$

$$
\frac{d m_{+}}{d t}=\tau\left(P_{-}^{+} p_{-\rightarrow+} m_{+-}-2 P_{+}^{-} p_{+\rightarrow-} m_{+}\right)+\rho n_{+} P_{+}^{-},
$$

$$
\frac{d \mu_{-}}{d t}=\mu_{-}\left(1-\mu_{-}-E_{-}\right)+\tau \frac{n_{+}}{n_{-}}\left(\mu_{+}-\mu_{-}\right) P_{+}^{-} p_{+\rightarrow-},
$$

$$
\frac{d \mu_{+}}{d t}=\mu_{+}\left(1-\mu_{+}-E_{+}\right)+\tau \frac{n_{-}}{n_{+}}\left(\mu_{-}-\mu_{+}\right) P_{-}^{+} p_{-\rightarrow+} .
$$


It is important to note that in most previous works on adaptive networks a closed set of macroscopic equations is obtained by assuming that links in the network are drawn at random and interactions take place between nodes that are connected by them $[6,33]$. In this work nodes, not links, are randomly drawn and initiate an interaction with neighboring nodes. This subtle difference changes the effective time scale of the system. Specifically, in our model only a maximum of $N$ out of all $M$ links are affected by interactions between nodes during the same time as all $M$ links would be considered if interactions take place by randomly drawing links in the network. In other words, in our model it takes $M / N$ times longer to achieve the same number of updates, as one would obtain by considering per-link interactions.

For the above system, the stable fixed point's $n_{-0}$ component can be obtained numerically for different combinations of $\phi$ and $T$ [Fig. 3(b)]. The results are again in good agreement with the numerical simulations and imply that for every choice of $T>0$ there actually exists an appropriate choice of $\phi \in\left[\phi_{c_{1}}, \phi_{c_{2}}\right]$ so all nodes are likely to adopt the effort level $E_{-}$. The lower bound of the optimal rewiring probability $\phi_{c_{1}}$ can be obtained by utilizing Eq. (21) and the linear relationship between $\phi_{c_{1}}$ and $T$ for a fixed rate of social updates $\tau$ that lead to imitation as given in Eq. (30). We thus find $\phi_{c_{1}}(T, \Delta E)=\phi_{c_{2}}\left(1-\frac{2-2 \Delta E^{2}}{1+\Delta E^{2}} T\right) \forall 0<T<\frac{1+\Delta E^{2}}{2-2 \Delta E^{2}}$ and $\phi_{c_{1}}(T, \Delta E)=0$ otherwise. The upper bound $\phi_{c_{2}}$ at which the network fragments is obtained from a numerical bifurcation analysis as $\phi_{c_{2}} \approx 0.89$. The result is in good agreement with previous findings on the fragmentation threshold in adaptive networks for similar average degree $\bar{k}$ [34,35]. We find, however, that the computed fragmentation threshold $\phi_{c_{2}}$ is larger than what is expected from the numerical simulations [Fig. 3(a)]. This can either be due to the fact that moment closure as well as mean-field approximations are known to provide only rough estimates of the fragmentation threshold [33] or because finite-size effects in the numerical simulations cause the system to fragment for smaller values of $\phi$ than it would be expected for the limiting case $N \rightarrow \infty$ that is considered in the macroscopic approximations. A more detailed study of the network fragmentation and the corresponding threshold $\phi_{c_{2}}$ is a subject of future research.

\section{Consistency between approximations}

To illustrate the consistency of the set of differential equations describing the static setting (16)-(18) and the adaptive case (59)-(63), we set $\phi=0$ in the latter, compute its fixed points numerically and compare them with the static setting's fixed points (21) and (22). Figures 5(a)-5(c) show the different components of the stable fixed points as a function of the control parameter $T$ for a fixed $\Delta E=0.5$. The components $n_{-0}, \mu_{+0}$, and $\mu_{-0}$ align perfectly well for the static and the adaptive case. The gray shaded area in Fig. 5(a) indicates the center manifold (28) for which the system's stability cannot be assessed by standard linear stability analysis. However, numerically integrating the set of differential equations yields the expected behavior of $n_{-}(0) \rightarrow 0$ as $T \rightarrow 0$. Figure 5(d) displays again the $n_{-0}$ component of the adaptive model's stable fixed point for $\phi=0$ and different combinations of $T$ and $\Delta E$. The results match those of Fig. 1(b). Hence, the
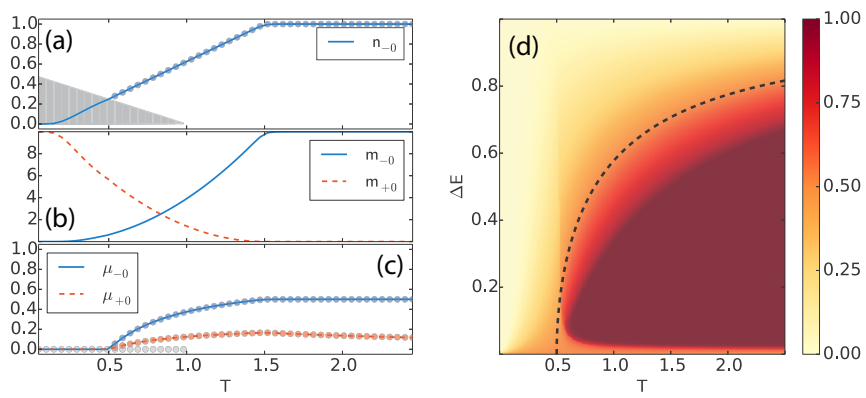

FIG. 5. (Color online) [(a)-(c)] The dependence of the adaptive (solid lines) and static model's (transparent scatter) stable fixed point on the expected waiting time $T$ for fixed parameters $\phi=0$ and $\Delta E=$ 0.5 . (d) The adaptive model's stable fixed point's $n_{-0}$ component indicating the fraction of nodes with effort $E_{-}$in the consensus state as a function of the two parameters $T$ and $\Delta E$ for $\phi=0$. The dashed line indicates the value of the critical waiting time $T_{c}$ obtained from the set of differential equations (16)-(18).

system of dynamic equations (59)-(63) can be interpreted as a consistent generalization of Eqs. (16)-(18).

\section{CONCLUSIONS}

We have introduced a model to describe emerging structure formation from the interplay of dynamics of and on networks manifested by the coevolution of social dynamics on the one hand and resource dynamics on the other hand. An adaptive voter model has been coupled to a set of logistic growth models, such that the state of the dynamic variables influences the imitation (i.e., social trait adoption) processes in the underlying social network which take place according to differences in harvest or payoff. We have derived rate equations for the system's macroscopic variables and demonstrated that the resulting system of differential equations yields stable fixed points which are in good agreement with the results from numerical simulations.

Our paradigmatic example illustrates that the interplay between both types of network dynamics gives rise to a variety of new phenomena, which have not been observed so far when only studying either of the two aspects. We have mainly found that the rate of interactions in the network determines the expected linear stability of the growth model's fixed points. However, for each choice of interaction rate there exists an appropriate range of the adaptive rewiring frequency so that the expected fraction of, e.g., nodes with effort $E_{-}$can be maximized. Notably, the subset of differential equations (59)(61) provides a general description of imitation and adaptation dynamics on a social network with binary states of nodes and symmetric imitation rules. Hence, it is applicable to study many other problems as long as the imitation probabilities $p_{-\rightarrow+}$ and $p_{+\rightarrow-}$, which do not have to be constant for all times, are chosen appropriately.

The proposed model also raises questions that need to be addressed in future research. In the course of the macroscopic approximation we have assumed all moments of higher order in stocks and network structure to vanish such that the set of differential equations could be closed. The results have been shown to be in good agreement with numerical simulations. 
However, a more in-depth analysis of whether the inclusion of higher-order moments would enable us to reproduce the steep transition between the two regimes of predominance of lowor high-effort nodes remains a relevant research questions. We also aim to estimate more thoroughly the critical waiting time $T_{c}$ at which the observed phase transition takes place and therefore investigate the expected time at which the low effort provides more harvest than the high effort given that no interaction between the nodes took place so far. Finally, we aim to obtain data from agricultural studies on, e.g., water usage or harvest exploitation of resources to test the findings and insights that we have obtained from our coevolutionary model with respect to real-world phenomena.

\section{ACKNOWLEDGMENTS}

This work was carried out within the framework of PIK's COPAN project. M.W. was supported by the German Federal Ministry for Science and Education via the BMBF Young Investigators Group CoSy-CC ${ }^{2}$ (Grant No. 01LN1306A). J.F.D. and W.L. acknowledge funding from the Stordalen Foundation (Norway) via the PB.net initiative and BMBF (project GLUES) and J.K. acknowledges the IRTG 1740 funded by Deutsche Forschungsgesellschaft (DFG) (Germany) and FAPESP. We thank R. V. Donner for helpful comments and suggestions on the manuscript and R. Grzondziel and C. Linstead for help with the IBM iDataPlex Cluster at the Potsdam Institute for Climate Impact Research.
[1] R. Albert and A.-L. Barabási, Statistical mechanics of complex networks, Rev. Mod. Phys. 74, 47 (2002).

[2] M. E. J. Newman, The structure and function of complex networks, SIAM Rev. 45, 167 (2003).

[3] T. Gross and B. Blasius, Adaptive coevolutionary networks: A review, J. R. Soc. Interface 5, 259 (2008).

[4] T. Gross and H. Sayama, Adaptive Networks (Springer, Berlin, 2009).

[5] P. Holme and J. Saramäki, Temporal networks, Phys. Rep. 519, 97 (2012).

[6] T. Gross, Carlos J. Dommar D’Lima, and B. Blasius, Epidemic dynamics on an adaptive network, Phys. Rev. Lett. 96, 208701 (2006).

[7] R. M. May and A. L. Lloyd, Infection dynamics on scale-free networks, Phys. Rev. E 64, 066112 (2001).

[8] R. Pastor-Satorras and A. Vespignani, Epidemic spreading in scale-free networks, Phys. Rev. Lett. 86, 3200 (2001).

[9] G. C. M. A. Ehrhardt, M. Marsili, and F. Vega-Redondo, Phenomenological models of socioeconomic network dynamics, Phys. Rev. E 74, 036106 (2006).

[10] P. Holme and M. E. J. Newman, Nonequilibrium phase transition in the coevolution of networks and opinions, Phys. Rev. E 74, 056108 (2006).

[11] L. E. Blume, The statistical mechanics of strategic interaction, Game Econ. Behav. 5, 387 (1993).

[12] G. Szabó and C. Tőke, Evolutionary prisoner's dilemma game on a square lattice, Phys. Rev. E 58, 69 (1998).

[13] A. Traulsen, D. Semmann, R. D. Sommerfeld, H.-J. Krambeck, and M. Milinski, Human strategy updating in evolutionary games, Proc. Natl. Acad. Sci. USA 107, 2962 (2010).

[14] P. Ji, Thomas K. DM. Peron, P. J. Menck, F. A. Rodrigues, and J. Kurths, Cluster explosive synchronization in complex networks, Phys. Rev. Lett. 110, 218701 (2013).

[15] A. Arenas, A. Díaz-Guilera, J. Kurths, Y. Moreno, and C. Zhou, Synchronization in complex networks, Phys. Rep. 469, 93 (2008).

[16] R. Perman, Natural Resource and Environmental Economics (Pearson Education, Harlow, 2003).

[17] H. J. Schellnhuber, Discourse: Earth System analysis-The scope of the challenge, in Earth System Analysis (Springer, Berlin, 1998), pp. 3-195.

[18] H. J. Schellnhuber, 'Earth system' analysis and the second Copernican revolution, Nature 402, C19 (1999).
[19] S. J. Lade, A. Tavoni, S. A. Levin, and M. Schlüter, Regime shifts in a social-ecological system, Theor. Ecol. 6, 359 (2013).

[20] J. M. Anderies, S. Carpenter, W. Steffen, and J. Rockström, The topology of non-linear global carbon dynamics: From tipping points to planetary boundaries, Environ. Res. Lett. 8, 044048 (2013).

[21] O. Kellie-Smith and P. M. Cox, Emergent dynamics of the climate-economy system in the Anthropocene, Phil. T. Roy. Soc. A 369, 868 (2011).

[22] J. Brander and M. Taylor, The simple economics of Easter Island: A Ricardo-Malthus model of renewable resource use, Am. Econ. Rev. 88, 119 (1998).

[23] S. Motesharrei, J. Rivas, and E. Kalnay, Human and nature dynamics (HANDY): Modeling inequality and use of resources in the collapse or sustainability of societies, Ecol. Econ. 101, 90 (2014).

[24] M. Schlüter, R. R. J. Mcallister, R. Arlinghaus, N. Bunnefeld, K. Eisenack, F. Hölker, E. Milner-Gulland, B. Müller, E. Nicholson, M. Quaas, and M. Stöven, New horizons for managing the environment: A review of coupled social-ecological systems modeling, Nat. Resour. Model. 25, 219 (2012).

[25] J. S. Lansing and J. N. Kremer, Emergent properties of Balinese water temple networks: Coadaptation on a rugged fitness landscape, Am. Anthropol. 95, 97 (1993).

[26] J. S. Lansing, M. P. Cox, S. S. Downey, M. A. Janssen, and J. W. Schoenfelder, A robust budding model of Balinese water temple networks, World Archaeol. 41, 112 (2009).

[27] J. S. Lansing, Perfect Order: Recognizing Complexity in Bali (Princeton University Press, Princeton, NJ, 2012).

[28] A. G. Sanfey, Social decision-making: Insights from game theory and neuroscience, Science 318, 598 (2007).

[29] H. Ebel and S. Bornholdt, Coevolutionary games on networks, Phys. Rev. E 66, 056118 (2002).

[30] F. A. Haight, Handbook of the Poisson Distribution (Wiley, New York, 1967).

[31] A. Vespignani, Modelling dynamical processes in complex socio-technical systems, Nat. Phys. 8, 32 (2012).

[32] C. Castellano and R. Pastor-Satorras, Thresholds for epidemic spreading in networks, Phys. Rev. Lett. 105, 218701 (2010).

[33] G. Demirel, F. Vazquez, G. A. Böhme, and T. Gross, Momentclosure approximations for discrete adaptive networks, Physica D 267, 68 (2014). 
[34] H. Silk, G. Demirel, M. Homer, and T. Gross, Exploring the adaptive voter model dynamics with a mathematical triple jump, New J. Phys. 16, 093051 (2014).
[35] G. A. Böhme and T. Gross, Analytical calculation of fragmentation transitions in adaptive networks, Phys. Rev. E 83, 035101 (2011). 